\title{
Prognostic and clinicopathological role of long non-coding RNA UCA1 in various carcinomas
}

\author{
Xiaoxiong Wang ${ }^{1,2, *}$, Fei Peng ${ }^{1,2, *}$, Liang Cheng ${ }^{3, *}$, Guang Yang ${ }^{1,2}$, Daming Zhang ${ }^{1,2}$, \\ Jiaqi Liu ${ }^{1,2}$, Xin Chen ${ }^{1,2}$, Shiguang Zhao ${ }^{1,2}$ \\ ${ }^{1}$ Department of Neurosurgery, The First Affiliated Hospital of Harbin Medical University, Nangang District, Harbin, Heilongjiang \\ Province, 150001, People's Republic of China \\ ${ }^{2}$ Institute of Brain Science, Harbin Medical University, Nangang District, Harbin, Heilongjiang Province, 150001, People's \\ Republic of China \\ ${ }^{3}$ College of Bioinformatics Science and Technology, Harbin Medical University, Nangang District, Harbin, Heilongjiang \\ Province, 150081, People's Republic of China \\ *These authors contributed equally to this work
}

Correspondence to: Shiguang Zhao, email: guangsz@hotmail.com

Keywords: long noncoding RNA (InCRNA), urothelial cancer associated 1 (UCA1), cancer, prognosis

Received: August 10, $2016 \quad$ Accepted: February 27, $2017 \quad$ Published: March 09, 2017

Copyright: Wang et al. This is an open-access article distributed under the terms of the Creative Commons Attribution License (CC-BY), which permits unrestricted use, distribution, and reproduction in any medium, provided the original author and source are credited.

ABSTRACT

Urothelial cancer associated 1 (UCA1) as an oncogenic long non-coding RNA (LncRNA) was aberrantly upregulated in various solid tumors. Numerous studies have demonstrated overexpression of UCA1 is an unfavorable prognostic indicator in cancer patients. This study aimed to further explore the prognosis role and clinical significance of UCA1 in cancer. Eligible studies were recruited by a systematic search in PubMed, Embase, Cochrane Library and Web of Science databases. A total of 19/16 studies with 1587/1291 cancer patients were included to evaluate the association between UCA1 expression and overall survival (OS) and clinicopathological factors of malignancies by computing hazard ratio (HR), odds ratios (OR) and confidence interval (CI). The meta-analysis indicated overexpression of UCA1 was significantly correlated with unexpected OS in patients with cancer (pooled HR $=1.85,95 \%$ CI 1.62-2.10, $p<0.001)$. There was also a significantly negative association between high level of UCA1 and poor grade cancer (pooled OR $=2.74,95 \%$ CI 2.04-3.70, $p<0.001$ ) and positive lymphatic metastasis (pooled OR $=2.43,95 \% \mathrm{CI} 1.72-3.41, p<0.001$ ). In conclusion, our study suggested that UCA1 was correlated with more advanced clinicopathological features and poor prognosis as a novel predictive biomarker of patients with various tumors.

\section{INTRODUCTION}

Non-coding RNAs (ncRNAs) encoded by the eukaryotic genome are considered as a large number of RNAs which are not translated into proteins. Indeed, more than $90 \%$ of noncoding RNAs used to be recognized as "biological noises" in transcription progression compared with about $3 \%$ protein-coding genes until the development of high-throughput sequencing technology and large-scale mapping of transcriptomes $[1,2]$. Based on structural features and biological functions, ncRNAs family has been further classified into housekeeping and regulatory ncRNAs. Regulatory ncRNAs upon nucleotide length are generally divided into two subgroups: (1) short ncRNAs shorter than
200 nucleotides such as microRNAs (miRNAs); (2) long noncoding RNAs (lncRNAs) longer than 200 nucleotides such as intergenic lncRNAs (lincRNAs) and circular RNAs (circRNAs) [3, 4]. Accumulating evidence have revealed the major transcriptional and post-transcriptional regulation roles of IncRNAs emerge in transcription factor recruitment, chromatin remodeling, histone modification, pre-mRNA splicing, molecular sponge and scaffold, which are involved in development of normal tissue or organ and carcinogenesis and aggression of diverse malignancies [5-8].

Urothelial cancer associated 1 also known as UCA1 is located on 19p13.12 encodes 3 isoforms (1.4, 2.2 and $2.7 \mathrm{~kb}$ ) with ployA tails, in which the $2.2 \mathrm{~kb}$ isoform is called cancer upregulated drug resistant (CUDR). 
Previous study has shown UCA1, a highly conserved nuclear-enriched IncRNA, is ubiquitous in different development stages of urinary system, productive system, digestive system and respiratory system [9]. In cancers, UCA1 as an oncogene exhibited regulatory mechanisms responsible for cell proliferation, invasion, metastasis, apoptosis, metabolism and chemoresistance [10]. Cheng et al. reported that downregulation of UCA1 impaired chemoresistance of non-small cell lung cancer cells with non-T790M to gefitinib by inhibition of the AKT/mTOR pathway [11]. Na et al. indicated knockdown of UCA1 inhibited cell proliferation and induced cell apoptosis by inactivation of KLF4-KRT6/13 cascade in prostate cancer [12]. Wang et al. showed that UCA1 as an endogenous sponge restored the negative effect of $\mathrm{miR}-216 \mathrm{~b}$ on the growth and metastasis of hepatocellular carcinoma cells through activating FGFR1/ERK signaling pathway [13]. $\mathrm{Hu}$ et al. revealed the $\mathrm{X}$ protein encoded by HBV increased UCA1 expression which inhibited cell apoptosis and promoted cell proliferation and carcinogenesis by $\mathrm{HBx}$ UCA1/EZH2-p27Kip1 signaling axis in hepatocellular carcinoma cells [14].

Furthermore, increasing interest has been focused on whether UCA1 acts as a diagnosis and prognosis biomarker in cancer detection and treatment. UCA1 was firstly characterized as an effective diagnosis biomarker in bladder cancer with a high sensitivity and specificity (80.9\% and 91.8\%) [15]. Meanwhile, growing subsequent evidence suggest that aberrant overexpression of UCA1 is associated with high risk of poor outcome or clinicopathological characteristics in breast cancer, colorectal cancer, bladder cancer, esophageal squamous cell carcinoma, epithelial ovarian cancer, gastric cancer, hepatocellular carcinoma, melanoma, non-small cell lung cancer, prostate cancer and tongue squamous cell carcinoma [11-13, 15-27]. Thus, it is necessary to certificate the potential correlation between UCA1 expression and malignancies by a comprehensive analysis. In this meta-analysis, we qualified and evaluated present studies to explore the association of UCA1 with prognostic and clinicopathological significance in patients with different types of carcinomas.

\section{RESULTS}

\section{Study selection and characteristics}

Upon an electrical search on PubMed, Embase, Cochrane Library and Web of Science databases, a total of 19 eligible OS papers including 1587 tissue specimens from 1774 records published from 2014 to 2017 were enrolled by a cautious searching strategy and full-text screening, which were based on inclusion and exclusion criteria in this meta-analysis (Figure 1). Participants in 19 OS studies were all Asian with 10 types of tumors including non-small cell lung carcinoma (NSCLC), gastric cancer (GC), colorectal cancer (CRC), esophageal squamous cell carcinoma (ESCC), prostate cancer (PC), hepatocellular cancer (HCC), epithelial ovarian cancer (EOC), endometrial cancer (EC), pancreatic cancer (PAC), and breast cancer (BC) (Supplementary Table 1). The main characteristics of retrieved OS studies were summarized in Table 1. Meanwhile, total 16 available clinicopathological studies containing 1291 tissue samples were collected to analyze the correlation between UCA1 level and clinicopathological data (clinical stage, tumor size, lymphatic and distant metastasis) shown in (Supplementary Table 2). And all clinicopathological studies were Asian with 8 kinds of tumors above except prostate and breast cancer. Quality evaluation based on reporting recommendations for tumor marker prognostic studies (REMARK) guideline reflected quality score ranged from $40 \%$ to $80 \%$ in (Supplementary Table 3). With inconsistent cut-off values due to different detection methods, the patients were separated into high and low level of UCA1 groups. HRs with 95\% CIs were extracted from multivariate analysis in 14 studies, univariate analysis in 5 studies (estimated effects collected from Kaplan-Meier survival curve in 3 studies).

\section{Association of UCA1 expression with overall survival in human cancers}

To assess the association between the UCA1 expression and OS of all cancer patients, a total of 1587 patients with HRs and 95\% CIs were included. A significant association was observed between high UCA1 level and poor OS in patients with all 10 types of cancer (pooled HR $=1.85,95 \%$ CI 1.62-2.10, $p<0.001$ ) and no obvious heterogeneity existed across 19 studies under a fixed effect model $\left(\mathrm{I}^{2}=0.0 \%, \mathrm{p}_{\mathrm{H}}=0.905\right)$ in Figure $2 \mathrm{~A}$.

The subgroup analysis in a fixed effect model, random effect model and meta-regression was conducted by cancer types, analysis methods, sample sizes and quality scores for a subsequent investigation of potential heterogeneity in Table 2 . It revealed a significant association between increased UCA1 and OS in patients with GC (pooled HR $=2.32,95 \%$ CI 1.61-3.33, $p<$ $0.001)$, CRC $($ pooled HR $=2.16,95 \%$ CI 1.29-3.62, $p=$ $0.004)$, HCC $($ pooled HR $=1.89,95 \%$ CI 1.20-3.00, $p=$ 0.007 ), and PAC (pooled HR $=1.60,95 \%$ CI 1.16-2.22, $p=0.004)$. There showed no statistical significance of heterogeneity test in subgroups of $\mathrm{GC}\left(\mathrm{I}^{2}=0.0 \%, \mathrm{p}_{\mathrm{H}}=\right.$ $0.928), \mathrm{CRC}\left(\mathrm{I}^{2}=0.0 \%, \mathrm{p}_{\mathrm{H}}=0.948\right), \mathrm{HCC}\left(\mathrm{I}^{2}=0.0 \%, \mathrm{p}_{\mathrm{H}}=\right.$ $0.899)$, and PAC $\left(\mathrm{I}^{2}=0.0 \%, \mathrm{p}_{\mathrm{H}}=0.457\right)$. Then, a significant effect of elevated UCA1 on OS emerged in multivariable analysis subgroup (pooled HR $=1.80,95 \%$ CI 1.55-2.09, $p<0.001$ ) and univariable analysis subgroup (pooled HR $=1.98,95 \%$ CI $1.55-2.55, p<0.001)$. No statistically significant heterogeneity was in multivariable analysis subgroup $\left(\mathrm{I}^{2}=0.0 \%, \mathrm{p}_{\mathrm{H}}=0.722\right)$ and univariable analysis subgroup $\left(\mathrm{I}^{2}=0.0 \%, \mathrm{p}_{\mathrm{H}}=0.956\right)$. Meanwhile, we also 
detected a significant correlation between overexpression of UCA1 and poor prognosis of cancer patients in large specimen size subgroup (pooled $\mathrm{HR}=1.68$, 95\% CI $1.41-2.00, p<0.001)$ and small specimen size subgroup (pooled HR $=2.07,95 \%$ CI 1.71-2.50, $p<0.001$ ) with no evident heterogeneity $\left(\mathrm{I}^{2}=0.0 \%, \mathrm{p}_{\mathrm{H}}=0.639\right.$ and $\left.\mathrm{I}^{2}=0.0 \%, \mathrm{p}_{\mathrm{H}}=0.979\right)$ respectively. To the quality score subgroups, we obtained similar association of upregulation of UCA1 with OS in high quality score subgroup (pooled $\mathrm{HR}=1.98,95 \%$ CI $1.58-2.48, p<0.001)$ and low quality score subgroup (pooled HR $=1.79,95 \%$ CI 1.53-2.09, $p<0.001$ ). No evidence of significant heterogeneity was found across 2 subgroups $\left(\mathrm{I}^{2}=0.0 \%, \mathrm{p}_{\mathrm{H}}=0.576\right.$ and $\mathrm{I}^{2}=$ $\left.0.0 \%, \mathrm{p}_{\mathrm{H}}=0.921\right)$. Quantified heterogeneous test by metaregression indicated that no specific factor accounted for the heterogeneity among studies of interest consistent with the outcomes of subgroup analysis in cancer type, analysis method, tumor sample size and quality score covariates.

Publication bias evaluated by funnel plot and Egger's test indicated that there was evident asymmetry in this meta-analysis ( $\mathrm{p}_{\text {Egger's }}=0.006$, Figure $2 \mathrm{~B}$ and $2 \mathrm{C}$ ). And publication bias of subgroup showed small-studies effects existed in large tumor sample size $\left(\mathrm{p}_{\text {Egger's }}=0.001\right)$, high quality score $\left(\mathrm{p}_{\text {Egger's }}<0.001\right)$ and multivariate analysis subgroup ( $\left.\mathrm{p}_{\text {Egger's }}<0.001\right)$ in (Supplementary Table 4). However, sensitivity analysis by removing each research in turn showed the residual pooled HRs of OS were not impacted dramatically in Figure 2D.

\section{Correlation between UCA1 level and clinical characteristics in patients with cancer}

In the clinicopathological studies, 16 researches consisting of 1291 tumor samples with a correlation between clinicopathological features and UCA1 expression were retrieved in OR analysis. Clinical stage, lymph node metastasis, distant metastasis and tumor size data were collected to analyze. UCA1 expression was significantly different in these clinicopathological factors (Supplementary Table 2). We found high UCA1 expression was associated with high grade cancer (pooled $\mathrm{OR}=2.74,95 \%$ CI 2.04-3.70, $p<0.001)$, positive lymphatic metastasis (pooled OR $=2.43,95 \%$ CI 1.72 $3.41, p<0.001$ ), and distant metastasis (pooled $\mathrm{OR}=2.10$, 95\% CI 1.13-3.89, $p<0.001)$ in Table 3-6.

The subgroup analysis stratified by cancer type, sample size and quality score uncovered the resource of heterogeneity under the fixed effect model, random

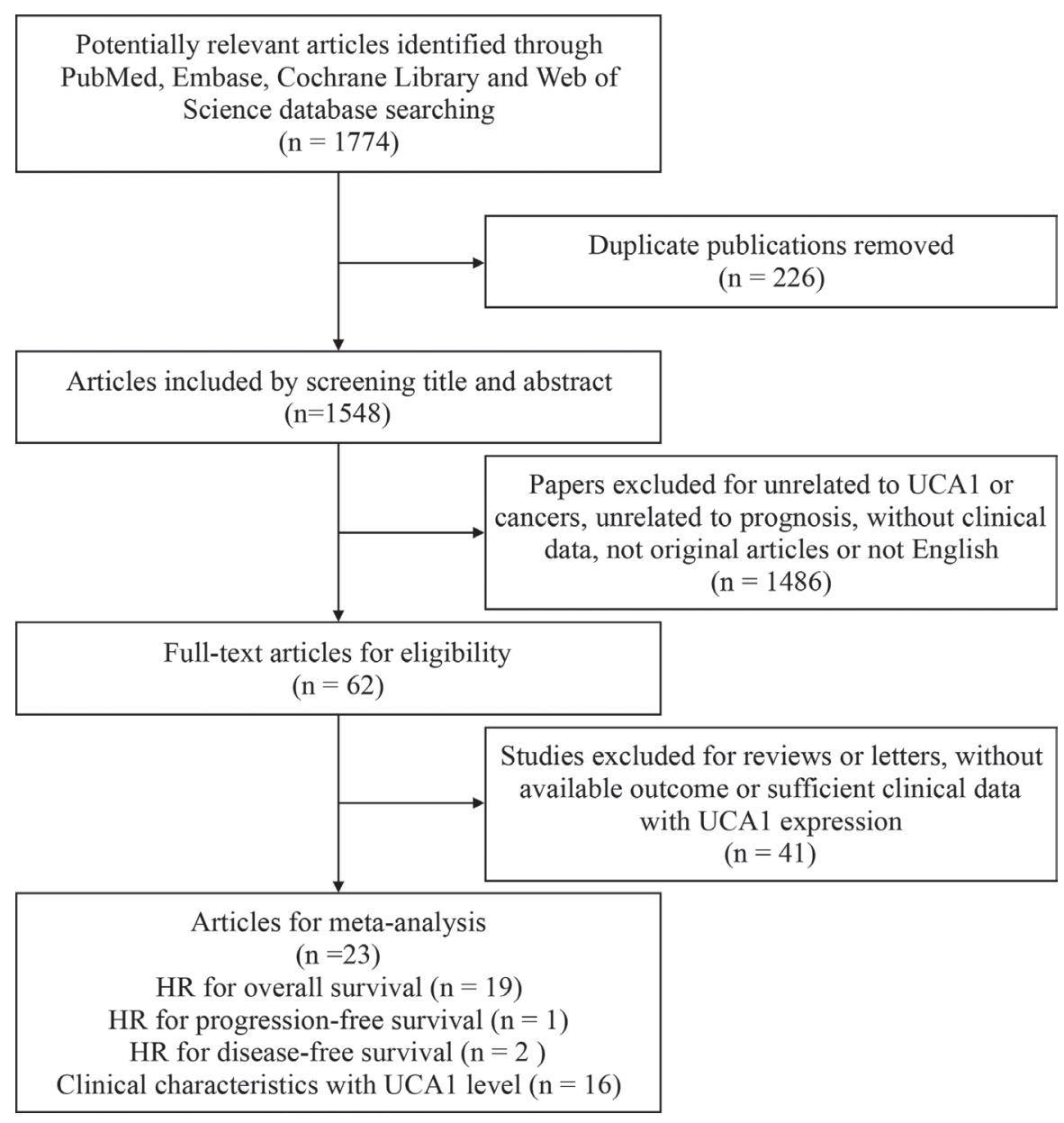

Figure 1: Workflow of searching strategy and study selection in the meta-analysis. 
Table 1: The main characteristics of included OS studies in the prognosis based on meta-analysis

\begin{tabular}{|c|c|c|c|c|c|c|c|c|c|c|}
\hline Study & Year & Region & $\begin{array}{l}\text { Tumor } \\
\text { type }\end{array}$ & $\begin{array}{c}\text { Sample } \\
\text { size }\end{array}$ & Specimen & Method & Cut-off value & Outcome & Analysis & $\begin{array}{c}\text { Quality } \\
\text { score (\%) }\end{array}$ \\
\hline $\mathrm{Li}$, et al. ${ }^{20}$ & 2014 & China & ESCC & 90 & Tissue & qRT-PCR & Mean (NG) & OS & Multivariable & 75 \\
\hline Zheng, et al. ${ }^{22}$ & 2015 & China & GC & 112 & Tissue & qRT-PCR & Median (17.24) & OS & Multivariable & 75 \\
\hline Wang, et al. ${ }^{13}$ & 2015 & China & NSCLC & 60 & Tissue & qRT-PCR & Median (NG) & OS & Multivariable & 75 \\
\hline $\mathrm{Ni}$, et al. ${ }^{17}$ & 2015 & China & $\mathrm{CRC}$ & 54 & Tissue & qRT-PCR & Median (NG) & OS & $\begin{array}{c}\text { Univariable } \\
\text { analysis }\end{array}$ & 60 \\
\hline $\mathrm{Na}$, et al. ${ }^{12}$ & 2015 & China & $\mathrm{PC}$ & 40 & Tissue & qRT-PCR & Median (NG) & OS & Univariable & 40 \\
\hline Gao, et al..$^{23}$ & 2015 & China & GC & 20 & Tissue & qRT-PCR & NG & OS & Multivariable & 40 \\
\hline $\mathrm{Nie}$, et al. ${ }^{26}$ & 2015 & China & NSCLC & 112 & Tissue & qRT-PCR & $\begin{array}{l}\text { Youden index } \\
\text { (NG) }\end{array}$ & OS & Multivariable & 70 \\
\hline Tao, et al. ${ }^{43}$ & 2015 & China & $\mathrm{CRC}$ & 80 & Tissue & qRT-PCR & $\begin{array}{l}\text { Upper quartile } \\
\text { (NG) }\end{array}$ & OS & Multivariable & 70 \\
\hline Wang, et al. ${ }^{13}$ & 2015 & China & $\mathrm{HCC}$ & 98 & Tissue & qRT-PCR & Median (NG) & OS & Multivariable & 70 \\
\hline Yang, et al. ${ }^{44}$ & 2015 & Korea & $\mathrm{HCC}$ & 240 & Tissue & microarray & Median (6.51) & OS & $\begin{array}{c}\text { Univariable } \\
\text { analysis }\end{array}$ & 50 \\
\hline Bian, et al. ${ }^{19}$ & 2016 & China & $\mathrm{CRC}$ & 90 & Tissue & qRT-PCR & Median (NG) & OS & Multivariable & 70 \\
\hline Yang, et al. ${ }^{21}$ & 2016 & China & EOC & 53 & Tissue & qRT-PCR & Median (NG) & OS & Multivariable & 75 \\
\hline Zhang, et al. ${ }^{45}$ & 2016 & China & EOC & 117 & Tissue & qRT-PCR & Median (NG) & OS & Multivariable & 70 \\
\hline $\mathrm{Lu}$, et al. ${ }^{46}$ & 2016 & China & $\mathrm{EC}$ & 45 & Tissue & qRT-PCR & Median (NG) & OS & Univariable & 65 \\
\hline Shang, et al. ${ }^{24}$ & 2016 & China & GC & 77 & Tissue & qRT-PCR & Median (NG) & OS & Multivariable & 75 \\
\hline Chen, et al. ${ }^{47}$ & 2016 & China & PAC & 128 & Tissue & qRT-PCR & Mean (NG) & OS & Multivariable & 80 \\
\hline $\mathrm{Fu}$, et al. ${ }^{48}$ & 2016 & China & PAC & 80 & Tissue & qRT-PCR & Median (NG) & OS & Multivariable & 80 \\
\hline Liu, et al. ${ }^{49}$ & 2016 & China & $\mathrm{BC}$ & 54 & Tissue & qRT-PCR & Median (NG) & OS & Univariable & 45 \\
\hline Zuo, et al. ${ }^{50}$ & 2017 & China & $\mathrm{GC}$ & 37 & Tissue & qRT-PCR & Median (NG) & OS & Multivariable & 75 \\
\hline
\end{tabular}

Abbreviations: ESCC, esophageal squamous cell carcinoma; GC, gastric cancer; NSCLC, non-small cell lung carcinoma; CRC, colorectal cancer; PC, prostate cancer; HCC, hepatocellular cancer; EOC, epithelial ovarian cancer; EC, endometrial cancer; PAC, pancreatic cancer; BC, breast cancer; qRT-PCR, quantitative real-time PCR; OS, overall survival; NG, not given.

effect model and meta-regression analysis (Table 3-6). There existed a significantly statistical heterogeneity across distant metastasis subgroup $\left(\mathrm{I}^{2}=56.5 \%, \mathrm{p}_{\mathrm{H}}=\right.$ $0.024)$ in 8 clinicopathological studies shown in Table 6. In distant metastasis heterogeneity analysis, we detected a significant heterogeneity in large sample size $\left(\mathrm{I}^{2}=71.9 \%, \mathrm{p}_{\mathrm{H}}=0.014\right)$ subgroup. No evidence of statistical heterogeneity was detected in cancer type and lymphatic metastasis subgroup $\left(\mathrm{I}^{2}=30.8 \%, \mathrm{p}_{\mathrm{H}}=0.116\right.$ and $\mathrm{I}^{2}=47.1 \%, \mathrm{p}_{\mathrm{H}}=0.023$, respectively). However, only CRC showed clinical significance and no obvious heterogeneity (pooled OR $=2.38,95 \%$ CI $1.18-4.80, p$ $\left.<0.001, \mathrm{I}^{2}=49.5 \%, \mathrm{p}_{\mathrm{H}}=0.114\right)$ by stratification analysis of cancer type. In subsequent stratification analysis of lymphatic metastasis, there was no evidence of significant heterogeneity in CRC (pooled OR $=2.07$, 95\% CI 1.25 $3.43, p=0.003, \mathrm{I}^{2}=5.6 \%, \mathrm{p}_{\mathrm{H}}=0.365$ ) and EOC (pooled $\mathrm{OR}=3.16,95 \%$ CI $1.59-6.27$, p $p<0.001, \mathrm{I}^{2}=0.0 \%$, $\left.\mathrm{p}_{\mathrm{H}}=0.684\right)$ subgroup.

A Egger's linear regression test was conducted to evaluate publication bias of these clinicopathological covariates. The result showed no publication bias existed between tumor size and distant metastasis subgroup in (Supplementary Table 5) $\left(\mathrm{p}_{\text {Egger's }}=0.622\right.$ and $\mathrm{p}_{\text {Egger's }}=0.653$, 
respectively) and no asymmetry among 4 subgroups in the sensitivity analysis (Supplementary Figure 1).

\section{DISCUSSION}

Cancer attacks all humankinds as a major cause of morbidity and mortality in worldwide regions with approximately 14.1 million new cancer cases and 8.2 million cancer-related deaths in 2012 [28]. Despite significant advances in cancer treatment, an unexpected long-term overall survival is still an important public health challenge. Therefore, novel strategies for detection of diagnostic and prognostic biomarkers and identification of potential therapeutic targets have attracted increasing interest.

The emerging next generation sequencing technology and large-scale of transcriptome mapping have provided an opportunity to facilitate to understand genome information and identify over 90\% non-coding RNAs regarded as "transcriptional noise" before. Continuing advances in these new technologies indicate the vital and complex functions of lncRNAs in gene regulation. With these updated views, the central dogma may be rewritten [6, 7]. Recent numerous studies have confirmed that lncRNAs as oncogenes or tumor suppressors play important regulatory roles in biological progress of a broad range of cancers or other human diseases. Substantial progress of lncRNAs demonstrate they may act as molecular scaffolds, sponges or co-activators by interaction with DNA, RNA or proteins in cancer nosogenesis $[1,5,6]$. Currently, a growing body of evidence revealed aberrant UCA1 as an oncogene in various malignancies [11-13, 15-27]. Intensive researches have showed overexpression of UCA1 can promote the progression of proliferation, invasion, migration, metastasis, chemoresistance in a variety of cancers [10]. Moreover, the dysregulation of UCA1 was also found in acute myocardial infarction, kidney damage and neurodegenerative diseases

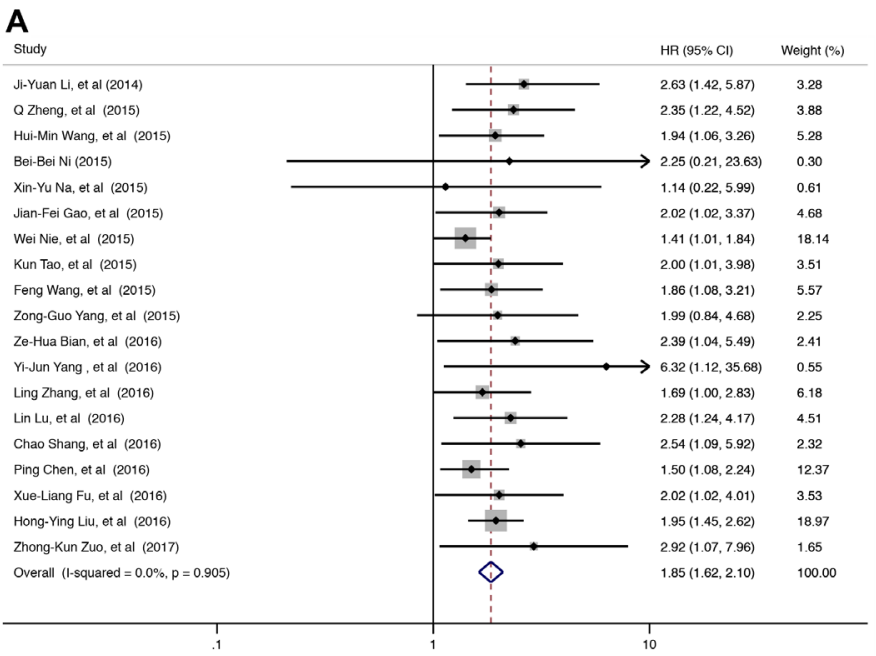

B
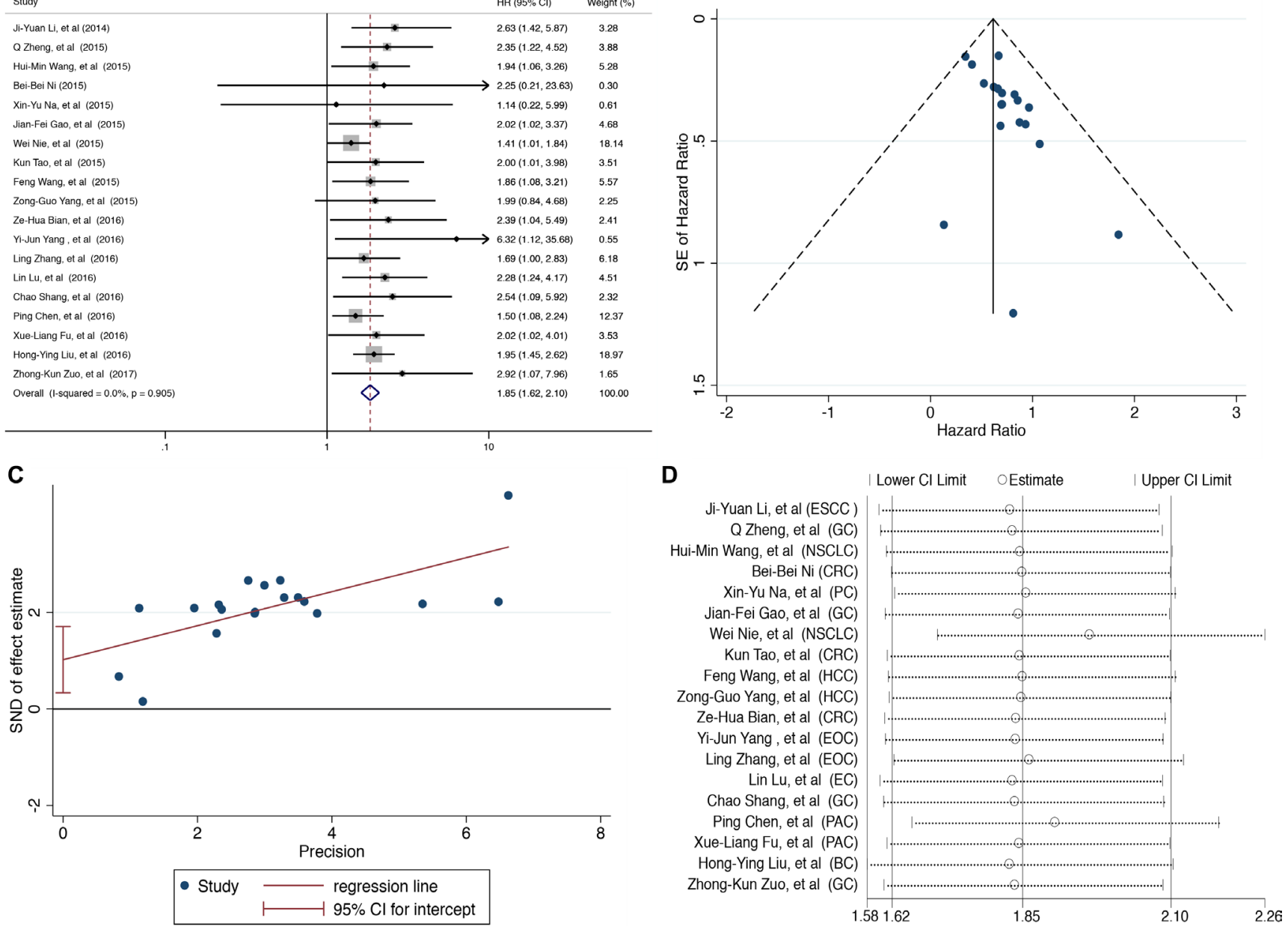

Figure 2: Prognostic value of UCA1 for OS of cancer patients. (A) Forest plot of HR studies of UCA1 for OS in a fixed effect model. Each study is represented by a square and the center of which denotes the HR with a horizontal 95\% Cis lines. The diamond shows the overall HR for combined results. Weights are from a fixed effect analysis. (B) Funnel plot for potential publication bias in OS analysis. Standard error (SE) of hazard ratio displays a measure of study size on the vertical axis against hazard ratio on the horizontal axis. (C) Egger's test for potential publication bias in OS analysis. Standard normal deviate (SND) is defined as the hazard ratio divided by its standard error which is regressed against the estimate's precision. (D) Sensitivity analysis of the effect of the individual study on the pooled HRs. 
Table 2: Subgroup and meta-regression analysis of HRs in different cancer type, analysis method, sample size and quality score subgroup

\begin{tabular}{|c|c|c|c|c|c|c|c|}
\hline \multirow{2}{*}{$\begin{array}{l}\text { Subgroup } \\
\text { analysis }\end{array}$} & \multirow{2}{*}{$\begin{array}{l}\text { No. of } \\
\text { studies }\end{array}$} & \multirow{2}{*}{$\begin{array}{c}\text { No. of } \\
\text { patients }\end{array}$} & \multicolumn{2}{|c|}{ Pool HR (95\% CI) } & \multirow{2}{*}{$\begin{array}{c}\text { Meta- } \\
\text { regression } \\
(p \text { value })\end{array}$} & \multicolumn{2}{|c|}{$\begin{array}{l}\text { Heterogeneity } \\
\text { (random) }\end{array}$} \\
\hline & & & Fixed & Random & & $I^{2}(\%)$ & $p$ value \\
\hline Overall survival & 19 & 1587 & $1.85(1.62-2.10)$ & $1.85(1.62-2.10)$ & - & 0.0 & 0.905 \\
\hline \multicolumn{8}{|l|}{ Cancer type } \\
\hline Digestive system & 12 & 1106 & $1.97(1.63-2.38)$ & $1.97(1.63-2.38)$ & - & 0.0 & 0.963 \\
\hline GC & 4 & 246 & $2.32(1.61-3.33)$ & $2.32(1.61-3.33)$ & 0.987 & 0.0 & 0.928 \\
\hline $\mathrm{CRC}$ & 3 & 224 & $2.16(1.29-3.62)$ & $2.16(1.29-3.62)$ & 0.817 & 0.0 & 0.948 \\
\hline $\mathrm{HCC}$ & 2 & 338 & $1.89(1.20-3.00)$ & $1.89(1.20-3.00)$ & 0.925 & 0.0 & 0.899 \\
\hline PAC & 2 & 208 & $1.60(1.16-2.22)$ & $1.60(1.16-2.22)$ & 0.240 & 0.0 & 0.457 \\
\hline ESCC & 1 & 90 & $2.63(1.29-5.35)$ & $2.63(1.29-5.35)$ & - & - & - \\
\hline Respiratory system & 2 & 172 & $1.51(1.16-1.98)$ & $1.51(1.16-1.98)$ & - & 0.0 & 0.328 \\
\hline NSCLC & 2 & 172 & $1.51(1.16-1.98)$ & $1.51(1.16-1.98)$ & 0.454 & 0.0 & 0.328 \\
\hline Reproductive system & 4 & 269 & $1.98(1.57-2.50)$ & $1.98(1.57-2.50)$ & - & 0.0 & 0.511 \\
\hline EOC & 2 & 170 & $1.88(1.15-3.09)$ & $2.50(0.77-8.13)$ & 0.883 & 51.2 & 0.152 \\
\hline $\mathrm{EC}$ & 1 & 45 & $2.28(1.24-4.18)$ & $2.28(1.24-4.18)$ & 0.961 & - & - \\
\hline $\mathrm{BC}$ & 1 & 54 & $1.95(1.45-2.62)$ & $1.95(1.45-2.62)$ & 0.795 & - & - \\
\hline Urinary system & 1 & 40 & $1.14(0.22-5.95)$ & $1.14(0.22-5.95)$ & - & - & - \\
\hline $\mathrm{PC}$ & 1 & 40 & $1.14(0.22-5.95)$ & $1.14(0.22-5.95)$ & 0.530 & - & - \\
\hline \multicolumn{8}{|l|}{ Analysis method } \\
\hline Multivariable analysis & 14 & 1154 & $1.80(1.55-2.09)$ & $1.80(1.55-2.09)$ & - & 0.0 & 0.722 \\
\hline Univariable analysis & 5 & 433 & $1.98(1.55-2.55)$ & $1.98(1.55-2.55)$ & 0.913 & 0.0 & 0.956 \\
\hline \multicolumn{8}{|l|}{ Sample size } \\
\hline Size $\geq 90$ & 8 & 987 & $1.68(1.41-2.00)$ & $1.68(1.41-2.00)$ & - & 0.0 & 0.639 \\
\hline Size $<90$ & 11 & 600 & $2.07(1.71-2.50)$ & $2.07(1.71-2.50)$ & 0.538 & 0.0 & 0.979 \\
\hline \multicolumn{8}{|l|}{ Quality scores } \\
\hline Score $\geq 75$ & 8 & 637 & $1.98(1.58-2.48)$ & $1.98(1.58-2.48)$ & - & 0.0 & 0.576 \\
\hline Score $<75$ & 11 & 950 & $1.79(1.53-2.09)$ & $1.79(1.53-2.09)$ & 0.296 & 0.0 & 0.921 \\
\hline
\end{tabular}

Abbreviations: ESCC, esophageal squamous cell carcinoma; GC, gastric cancer; NSCLC, non-small cell lung carcinoma; CRC, colorectal cancer; PC, prostate cancer; HCC, hepatocellular cancer; EOC, epithelial ovarian cancer; EC, endometrial cancer; PAC, pancreatic cancer; BC, breast cancer.

[10]. In cancer, the binding between UCA1 promoter core region and transcription factors or complex $(\mathrm{C} / \mathrm{EBP} \alpha$, Ets-2, TAZ/YAP/TEAD and SMAD2/3) can enhance UCA1 promoter activity and gene expression [29, 30]. The upregulation of UCA1 is responsible for tumor cell proliferation by suppressing G0/G1 cell cycle arrest and apoptosis. Furthermore, increased UCA1 promotes cancer invasion and metastasis by activation of metastasis genes including MMP14, FGFR1/ERK and ZEB1/2-FSCN1, and enhances chemoresistance by a set of anti-apoptosis genes and signaling pathways (PARP/BCL-2, CREB1/ BCL-2/RAB22A, AKT/mTOR and Wnt signaling pathway) $[11,13,19,21,24,31-33]$. UCA1 is also a key molecular sponge or competing endogenous RNA (ceRNA) for miR1, miR-204-5p, miR-193a-3p, miR-145 and miR-216 $[13,19,26,32,34]$. Simultaneously, UCA1 is associated with clinical parameters and prognosis of cancer patients and may be a potential diagnosis biomarker in 
Table 3: The subgroup and meta-regression analysis of the association and heterogeneity between high UCA1 expression and clinical stage

\begin{tabular}{|c|c|c|c|c|c|c|c|}
\hline \multirow[t]{2}{*}{$\begin{array}{l}\text { Subgroup } \\
\text { analysis }\end{array}$} & \multirow[t]{2}{*}{$\begin{array}{l}\text { No. of } \\
\text { studies }\end{array}$} & \multirow[t]{2}{*}{$\begin{array}{c}\text { No. of } \\
\text { patients }\end{array}$} & \multicolumn{2}{|c|}{ Pool OR (95\% CI) } & \multirow{2}{*}{$\begin{array}{c}\text { Meta- } \\
\text { regression } \\
(p \text { value })\end{array}$} & \multicolumn{2}{|c|}{$\begin{array}{l}\text { Heterogeneity } \\
\text { (random) }\end{array}$} \\
\hline & & & Fixed & Random & & $I^{2}(\%)$ & $p$ value \\
\hline $\begin{array}{l}\text { ORs of tumor stage } \\
\text { subgroup }\end{array}$ & 16 & 1291 & $2.63(2.07-3.33)$ & $2.74(2.04-3.70)$ & - & 30.8 & 0.116 \\
\hline \multicolumn{8}{|l|}{ Cancer type } \\
\hline Digestive system & 11 & 909 & $2.45(1.86-3.24)$ & $2.61(1.74-3.91)$ & - & 47.4 & 0.04 \\
\hline $\mathrm{GC}$ & 2 & 149 & $3.32(1.66-6.66)$ & $4.18(1.10-16.0)$ & 0.855 & 61.2 & 0.109 \\
\hline $\mathrm{CRC}$ & 4 & 304 & $2.23(1.38-3.60)$ & $2.38(1.18-4.80)$ & 0.616 & 49.5 & 0.114 \\
\hline $\mathrm{HCC}$ & 2 & 158 & $2.78(1.46-5.30)$ & $2.53(0.56-11.4)$ & 0.702 & 79.9 & 0.026 \\
\hline PAC & 2 & 208 & $1.68(0.91-3.09)$ & $1.97(0.66-5.89)$ & 0.569 & 54.2 & 0.140 \\
\hline ESCC & 1 & 90 & $3.64(1.49-8.88)$ & $3.64(1.49-8.88)$ & 0.851 & - & - \\
\hline Respiratory system & 2 & 172 & $3.74(1.82-7.70)$ & $3.71(1.79-7.69)$ & - & 0.0 & 0.368 \\
\hline NSCLC & 2 & 172 & $3.74(1.82-7.70)$ & $3.71(1.79-7.69)$ & 0.869 & 0.0 & 0.368 \\
\hline $\begin{array}{l}\text { Reproductive } \\
\text { system }\end{array}$ & 3 & 210 & $2.86(1.60-5.14)$ & $2.88(1.60-5.17)$ & - & 0.0 & 0.730 \\
\hline EOC & 2 & 168 & $2.66(1.42-5.00)$ & $2.66(1.42-5.00)$ & 0.704 & 0.0 & 0.648 \\
\hline $\mathrm{EC}$ & 1 & 42 & $4.64(0.98-22.0)$ & $4.64(0.98-22.0)$ & - & - & - \\
\hline \multicolumn{8}{|l|}{ Sample size } \\
\hline Size $\geq 90$ & 7 & 745 & $2.50(1.84-3.40)$ & $2.53(1.80-3.55)$ & - & 15.7 & 0.310 \\
\hline Size $<90$ & 9 & 546 & $2.83(1.94-4.12)$ & $3.12(1.84-5.29)$ & 0.761 & 44.2 & 0.074 \\
\hline \multicolumn{8}{|l|}{ Quality scores } \\
\hline Score $\geq 75$ & 7 & 560 & $2.79(1.94-4.00)$ & $3.07(1.89-5.00)$ & - & 37.8 & 0.141 \\
\hline Score $<75$ & 9 & 731 & $2.51(1.83-3.45)$ & $2.56(1.72-3.82)$ & 0.768 & 32.8 & 0.156 \\
\hline
\end{tabular}

Abbreviations: ESCC, esophageal squamous cell carcinoma; GC, gastric cancer; NSCLC, non-small cell lung carcinoma; CRC, colorectal cancer; HCC, hepatocellular cancer; EOC, epithelial ovarian cancer; EC, endometrial cancer; PAC, pancreatic cancer.

gastric cancer, hepatocellular cancer and bladder cancer $[15,22,23,35]$.

Here, we performed this meta-analysis to explore the correlation between high expression of UCA1 and clinicopathological characteristics and evaluate the prognosis role of UCA1 for cancer patients. All of these results above suggest that high UCA1 expression may be regarded as an unfavorable predictor in different cancers (pooled HR $=1.85,95 \%$ CI 1.62-2.10, $p<$ 0.001). Meanwhile, the pooled data of eligible studies also indicated that high UCA1 expression was significant correlated with poor grade cancer (pooled $\mathrm{OR}=2.74,95 \%$ CI 2.04-3.70, $\left.p<0.001, \mathrm{I}^{2}=49.5 \%, \mathrm{p}_{\mathrm{H}}=0.1\right)$ and positive lymph node metastasis (pooled OR $=2.43,95 \%$ CI 1.72 $3.41, p<0.001)$.

Otherwise, it should be acknowledged that several limitations existed in this current meta-analysis. First, almost all available studies were performed in China. The prognostic role of UCA1 should be taken cautiously in other regions and ethnicities. Second, inadequate data from some types of cancer and subgroup analysis in HRs and ORs may be the origin of heterogeneity. Third, the inconsistent cutoff value of UCA1 expression due to different methods and criteria may result in some heterogeneity. Finally, included papers were only English and most of which reported positive results, which may generate publication bias. Thus, the predictive significance of evaluated UCA1 in poor prognosis of patients with cancer might be overestimated to some extent. In addition, recent publications demonstrated a friendly and open user server by computational approaches will facilitate novel technologies and findings accessible to the public and enhance their impacts [36-38]. Hence, we appeal to establish a web-server of raw and integrated data for further analysis in drug design 
Table 4: The subgroup and meta-regression analysis of the association and heterogeneity between high UCA1 expression and tumor size

\begin{tabular}{|c|c|c|c|c|c|c|c|}
\hline \multirow{2}{*}{$\begin{array}{l}\text { Subgroup } \\
\text { analysis }\end{array}$} & \multirow{2}{*}{$\begin{array}{l}\text { No. of } \\
\text { studies }\end{array}$} & \multirow{2}{*}{$\begin{array}{c}\text { No. of } \\
\text { patients }\end{array}$} & \multicolumn{2}{|c|}{ Pool OR (95\% CI) } & \multirow{2}{*}{$\begin{array}{c}\text { Meta- } \\
\text { regression } \\
(p \text { value })\end{array}$} & \multicolumn{2}{|c|}{$\begin{array}{l}\text { Heterogeneity } \\
\text { (random) }\end{array}$} \\
\hline & & & Fixed & Random & & $I^{2}(\%)$ & $p$ value \\
\hline $\begin{array}{l}\text { ORs of tumor size } \\
\text { subgroup }\end{array}$ & 13 & 1090 & $1.47(1.15-1.87)$ & $1.45(0.99-2.14)$ & - & 57.0 & 0.006 \\
\hline \multicolumn{8}{|l|}{ Cancer type } \\
\hline Digestive system & 9 & 762 & $1.41(1.06-1.88)$ & $1.42(0.66-2.37)$ & - & 64.6 & 0.004 \\
\hline $\mathrm{GC}$ & 2 & 149 & $0.71(0.37-1.35)$ & $1.01(0.17-6.04)$ & 0.603 & 81.5 & 0.02 \\
\hline $\mathrm{CRC}$ & 3 & 250 & $1.89(1.11-3.22)$ & $1.81(0.67-4.90)$ & 0.822 & 69.5 & 0.038 \\
\hline $\mathrm{HCC}$ & 2 & 155 & $1.57(0.83-2.96)$ & $1.47(0.55-3.93)$ & 0.667 & 55.2 & 0.135 \\
\hline PAC & 2 & 208 & $1.58(0.92-2.73)$ & $1.49(0.60-3.69)$ & - & 61.3 & 0.108 \\
\hline Respiratory system & 2 & 172 & $1.98(1.04-3.76)$ & $1.99(1.04-3.78)$ & - & 0.0 & 0.350 \\
\hline NSCLC & 2 & 172 & $1.98(1.04-3.76)$ & $1.99(1.04-3.78)$ & 0.938 & 0.0 & 0.350 \\
\hline $\begin{array}{l}\text { Reproductive } \\
\text { system }\end{array}$ & 2 & 156 & $1.36(0.73-2.54)$ & $1.19(0.34-4.18)$ & - & 71.6 & 0.060 \\
\hline EOC & 2 & 156 & $1.36(0.73-2.54)$ & $1.19(0.34-4.18)$ & 0.623 & 71.6 & 0.060 \\
\hline \multicolumn{8}{|l|}{ Sample size } \\
\hline Size $\geq 90$ & 6 & 643 & $1.76(1.28-2.41)$ & $1.83(1.02-3.28)$ & - & 69.1 & 0.006 \\
\hline Size $<90$ & 7 & 447 & $1.13(0.77-1.66)$ & $1.13(0.71-1.80)$ & 0.575 & 29.5 & 0.203 \\
\hline \multicolumn{8}{|l|}{ Quality scores } \\
\hline Score $\geq 75$ & 6 & 470 & $1.07(0.75-1.54)$ & $1.08(0.58-2.00)$ & - & 61.2 & 0.024 \\
\hline Score $<75$ & 7 & 620 & $1.92(1.38-2.68)$ & $1.88(1.23-2.89)$ & 0.599 & 37.9 & 0.140 \\
\hline
\end{tabular}

Abbreviations: GC, gastric cancer; NSCLC, non-small cell lung carcinoma; CRC, colorectal cancer; HCC, hepatocellular cancer; EOC, epithelial ovarian cancer; PAC, pancreatic cancer.

and in-depth investigation, such as UCA1. In conclusion, the present analysis showed that overexpression of UCA1 might predict a poor prognosis in various types of malignancies, especially in Chinese population and was associated with poor cancer stage and positive lymphatic metastasis. Therefore, UCA1 may serve as an effective biomarker to predict prognosis and tumor progression of patients with cancer. Nevertheless, more large-scale and well-designed studies are required to update the findings of this analysis.

\section{MATERIALS AND METHODS}

\section{Search strategy and study selection criteria}

Systematic literature searches were conducted in PubMed, Embase, Cochrane Library and Web of Science databases (up to January 17, 2017) with the following searching strategy: "UCA1" OR "UCA 1" OR "UCA1" OR "urothelial cancer associated 1" OR "urothelial cancer associated-1" OR "CUDR" OR "LINC00178" OR "NCRNA00178" OR “onco-lncRNA-36" OR "ENSG00000214049") AND ("cancer" OR "tumor"
OR "tumour" OR "carcinoma” OR "neoplasm" OR "adenoma" OR "sarcoma"). We manually searched retrieved references for potentially missing literatures. The cited articles were excluded from duplicated firstly, then titles and abstracts were carefully scanned to eliminate irrelevant studies. Finally, we prudently reviewed full texts of potential retrieved studies. Studies were available upon the eligibility criteria: (1) showed the relationship between the dichotomous UCA1 levels and prognosis and clinicopathological characteristics of patients with any type of cancer; (2) calculated HRs and 95\% CIs for overall survival (OS), progression-free survival (PFS), disease-free survival (DFS); (3) were published in English. Duplicated, non-dichotomous expression of UCA1 or non-English articles, absence of key survival outcome such as HRs, 95\% CIs or Kaplan-Meier survival curve, reviews, letters, laboratory studies of non-human research or comments were omitted.

\section{Data extraction}

Eligible data were deliberatively judged and double checked from available studies based on criteria 
Table 5: The subgroup and meta-regression analysis of the association and heterogeneity between high UCA1 expression and lymph node metastasis

\begin{tabular}{|c|c|c|c|c|c|c|c|}
\hline \multirow{2}{*}{$\begin{array}{l}\text { Subgroup } \\
\text { analysis }\end{array}$} & \multirow{2}{*}{$\begin{array}{l}\text { No. of } \\
\text { studies }\end{array}$} & \multirow{2}{*}{$\begin{array}{c}\text { No. of } \\
\text { patients }\end{array}$} & \multicolumn{2}{|c|}{ Pool OR (95\% CI) } & \multirow{2}{*}{$\begin{array}{l}\text { Meta- } \\
\text { regression } \\
(p \text { value })\end{array}$} & \multicolumn{2}{|c|}{$\begin{array}{l}\text { Heterogeneity } \\
\text { (random) }\end{array}$} \\
\hline & & & Fixed & Random & & $I^{2}(\%)$ & $p$ value \\
\hline $\begin{array}{l}\text { ORs of lymph node } \\
\text { metastasis subgroup }\end{array}$ & 15 & 1229 & $2.26(1.78-2.86)$ & $2.43(1.72-3.41)$ & - & 47.1 & 0.023 \\
\hline \multicolumn{8}{|l|}{ Cancer type } \\
\hline Digestive system & 10 & 849 & $2.05(1.55-2.71)$ & $2.17(1.42-3.31)$ & - & 52.1 & 0.027 \\
\hline $\mathrm{GC}$ & 2 & 149 & $1.36(0.71-2.60)$ & $1.99(0.32-12.5)$ & 0.290 & 81.0 & 0.022 \\
\hline $\mathrm{CRC}$ & 4 & 304 & $2.07(1.28-3.34)$ & $2.07(1.25-3.43)$ & 0.290 & 5.6 & 0.365 \\
\hline $\mathrm{HCC}$ & 1 & 98 & $5.46(2.26-13.2)$ & $5.46(2.26-13.2)$ & 0.970 & - & - \\
\hline PAC & 2 & 208 & $1.48(0.85-2.57)$ & $1.46(0.78-2.73)$ & 0.235 & 19.6 & 0.265 \\
\hline ESCC & 1 & 90 & $3.57(1.48-8.74)$ & $3.57(1.48-8.74)$ & 0.595 & - & - \\
\hline Respiratory system & 2 & 172 & $\begin{array}{r}2.23 \\
(1.17-4.25)\end{array}$ & $2.54(0.70-9.23)$ & - & 71.1 & 0.063 \\
\hline NSCLC & 2 & 172 & $2.23(1.17-4.25)$ & $2.54(0.70-9.23)$ & 0.372 & 71.1 & 0.063 \\
\hline Reproductive system & 3 & 208 & $3.65(1.96-6.81)$ & $3.70(1.98-6.91)$ & - & 0.0 & 0.509 \\
\hline EOC & 2 & 163 & $3.16(1.59-6.27)$ & $3.16(1.59-6.27)$ & 0.495 & 0.0 & 0.684 \\
\hline $\mathrm{EC}$ & 1 & 45 & $7.84(1.77-34.8)$ & $7.84(1.77-34.8)$ & 0.961 & - & - \\
\hline \multicolumn{8}{|l|}{ Sample size } \\
\hline Size $\geq 90$ & 7 & 740 & $2.09(1.55-2.83)$ & $2.14(1.36-3.53)$ & - & 52.5 & 0.049 \\
\hline Size $<90$ & 8 & 489 & $2.55(1.74-3.73)$ & $2.89(1.67-5.00)$ & 0.589 & 46.8 & 0.068 \\
\hline \multicolumn{8}{|l|}{ Quality scores } \\
\hline Score $\geq 75$ & 7 & 560 & $2.02(1.43-2.84)$ & $2.27(1.29-4.00)$ & - & 58.9 & 0.024 \\
\hline Score $<75$ & 8 & 669 & $2.51(1.81-3.49)$ & $2.60(1.69-4.00)$ & 0.597 & 36.9 & 0.135 \\
\hline
\end{tabular}

Abbreviations: ESCC, esophageal squamous cell carcinoma; GC, gastric cancer; NSCLC, non-small cell lung carcinoma; CRC, colorectal cancer; HCC, hepatocellular cancer; EOC, epithelial ovarian cancer; EC, endometrial cancer; PAC, pancreatic cancer.

of inclusion and exclusion. To each study, we carefully extracted the following information: first author, journal, year of publication, country, ethnicity of the study population, type of specimen, carcinoma type, number of patients, detection method, cut-off value, followup, quality score and HR and $95 \%$ CI for OS, PFS, or DFS. Quality assessment of the available studies was performed upon the REMARK guideline [39]. HR, as a dominant indicator of interest, was respectively extracted from multivariable analysis, univariate analysis, additional information of first authors' e-mails and estimated from graphical survival plots by Engauge Digitizer 4.1 software as described previously, if only Kaplan-Meier curve existed [40, 41]. Clinical parameters with dichotomous UCA1 levels also were retrieved such as clinical stage (TNM stage), tumor size, lymphatic and distant metastasis.

\section{Statistical analysis}

All analysis was conducted with STATA software version 12.0 (STATA Corporation, College Station, TX, USA). Odds Ratios (ORs) were performed to analyze the association of UCA1 expression with clinical stage, tumor size, lymphatic and distant metastasis and HRs with corresponding $95 \%$ CIs were utilized to estimate the relationship strength between UCA1 expression and patients' prognosis. If the HRs were not directly reported in original articles, we calculated the essential data upon the previously reported methods [40]. The pooled HRs were estimated using a fixed effect model (Mantel-Haenszel) in the absence of heterogeneity, if heterogeneity was observed applying a random effect model (DerSimoian-Laird) and meta-regression analysis $[41,42]$. The heterogeneity tests of combined HRs and ORs were carried out by Cochran's $Q$ test and Higgins 
Table 6: The subgroup and meta-regression analysis of the association and heterogeneity between high UCA1 expression and distant metastasis

\begin{tabular}{|c|c|c|c|c|c|c|c|}
\hline \multirow{2}{*}{$\begin{array}{l}\text { Subgroup } \\
\text { analysis }\end{array}$} & \multirow{2}{*}{$\begin{array}{l}\text { No. of } \\
\text { studies }\end{array}$} & \multirow{2}{*}{$\begin{array}{l}\text { No. of } \\
\text { patients }\end{array}$} & \multicolumn{2}{|c|}{ Pool OR (95\% CI) } & \multirow{2}{*}{$\begin{array}{c}\text { Meta- } \\
\text { regression } \\
(p \text { value })\end{array}$} & \multicolumn{2}{|c|}{$\begin{array}{l}\text { Heterogeneity } \\
\text { (random) }\end{array}$} \\
\hline & & & Fixed & Random & & $\overline{I^{2}(\%)}$ & $p$ value \\
\hline $\begin{array}{c}\text { ORs of distant } \\
\text { metastasis subgroup }\end{array}$ & 8 & 687 & $2.05(1.42-2.96)$ & $2.10(1.13-3.89)$ & - & 56.5 & 0.024 \\
\hline \multicolumn{8}{|l|}{ Cancer type } \\
\hline Digestive system & 7 & 642 & $1.89(1.29-2.78)$ & $1.84(0.97-3.49)$ & - & 55.7 & 0.035 \\
\hline $\mathrm{GC}$ & 1 & 112 & $6.25(1.48-26.4)$ & $6.25(1.48-26.4)$ & - & - & - \\
\hline $\mathrm{CRC}$ & 3 & 224 & $2.08(0.97-4.48)$ & $1.98(0.85-4.59)$ & - & 8.0 & 0.337 \\
\hline $\mathrm{HCC}$ & 1 & 98 & $5.46(2.26-13.2)$ & $5.46(2.26-13.2)$ & - & - & - \\
\hline PAC & 2 & 208 & $1.66(0.86-3.21)$ & $1.66(0.85-3.22)$ & - & 0.0 & 0.446 \\
\hline Reproductive system & 1 & 45 & $6.25(1.48-26.4)$ & $6.25(1.48-26.4)$ & - & - & - \\
\hline EC & 1 & 45 & $6.25(1.48-26.4)$ & $6.25(1.48-26.4)$ & - & - & - \\
\hline \multicolumn{8}{|l|}{ Sample size } \\
\hline Size $\geq 90$ & 4 & 428 & $1.98(1.28-3.07)$ & $1.96(0.81-4.75)$ & - & 71.9 & 0.014 \\
\hline Size $<90$ & 4 & 259 & $2.22(1.12-4.42)$ & $2.34(0.86-6.37)$ & 0.752 & 44.3 & 0.143 \\
\hline \multicolumn{8}{|l|}{ Quality scores } \\
\hline Score $\geq 75$ & 3 & 320 & $1.20(0.71-2.10)$ & $1.14(0.55-2.36)$ & - & 38.4 & 0.197 \\
\hline Score $<75$ & 11 & 367 & $3.43(2.01-5.85)$ & $3.38(1.69-6.74)$ & 0.104 & 31.4 & 0.212 \\
\hline
\end{tabular}

Abbreviations: GC, gastric cancer; CRC, colorectal cancer; HCC, hepatocellular cancer; EC, endometrial cancer; PAC, pancreatic cancer.

I-squared statistic $\left(\mathrm{P}_{\mathrm{H}}<0.1\right.$ and $\left.\mathrm{I}^{2}>50 \%\right)$. To further explore the potential heterogeneity factors and outcome stability in studies, a subgroup analysis and a sensitivity analysis were utilized respectively in the meta-analysis. Publication bias was evaluated by a funnel plot and Egger's linear regression test with a significant publication bias by $P<0.05$.

\section{Abbreviations}

non-coding RNAs (ncRNAs), long non-coding RNA (LncRNA), non-coding RNAs (ncRNAs), overall survival (OS), progression-free survival (PFS), disease-free survival (DFS), hazard ratio (HR), odds ratios (OR), confidence interval (CI), microRNAs (miRNAs), intergenic lncRNAs (lincRNAs), circular RNAs (circRNAs), non-small cell lung carcinoma (NSCLC), gastric cancer (GC), colorectal cancer (CRC), esophageal squamous cell carcinoma (ESCC), prostate cancer (PC), hepatocellular cancer (HCC), epithelial ovarian cancer (EOC), endometrial cancer (EC), pancreatic cancer (PAC), breast cancer (BC), reporting recommendations for tumor marker prognostic studies (REMARK)

\section{Authors' Contributions}

SZ, XW, FP, and LC conceived and designed the study, coordinated the study and directed its implementation. XW and FP searched the publications, analyzed the data and wrote the manuscript. GY, DZ, JL and $\mathrm{XC}$ extracted the data, judged and checked results of data synthesis and made figures and tables. All authors reviewed and approved the final manuscript.

\section{ACKNOWLEDGMENTS AND FUNDING}

The work was supported by Natural Science Foundation of China (81472368 to SZ) and Scientific Fund of Heilongjiang Province for Youth (QC2016124 to XC).

\section{CONFLICTS OF INTEREST}

The authors declare no competing financial interests.

\section{REFERENCES}

1. Esteller M. Non-coding RNAs in human disease. Nature Reviews Genetics. 2011; 12:861-74. doi: 10.1038/nrg3074.

2. Consortium EP. An integrated encyclopedia of DNA elements in the human genome. Nature. 2012; 489:57-74. doi: 10.1038/nature11247.

3. Nagano T, Fraser P. No-nonsense functions for long noncoding RNAs. Cell. 2011; 145:178-81. doi: 10.1016/j. cell.2011.03.014.

4. Ponting CP, Oliver PL, Reik W. Evolution and functions of long noncoding RNAs. Cell. 2009; 136:629-41. doi: 10.1016/j.cell.2009.02.006. 
5. Yang L, Froberg JE, Lee JT. Long noncoding RNAs: fresh perspectives into the RNA world. Trends Biochem Sci. 2014; 39:35-43. doi: 10.1016/j.tibs.2013.10.002.

6. Gutschner T, Diederichs S. The hallmarks of cancer: a long non-coding RNA point of view. RNA Biol. 2012; 9:703-19. doi: 10.4161/rna.20481.

7. Shi X, Sun M, Liu H, Yao Y, Song Y. Long non-coding RNAs: a new frontier in the study of human diseases. Cancer Lett. 2013; 339:159-66. doi: 10.1016/j.canlet.2013.06.013.

8. Huang Y, Liu N, Wang JP, Wang YQ, Yu XL, Wang ZB, Cheng XC, Zou Q. Regulatory long non-coding RNA and its functions. J Physiol Biochem. 2012; 68:611-8. doi: 10.1007/s13105-012-0166-y.

9. Wang F, Li X, Xie X, Zhao L, Chen W. UCA1, a nonprotein-coding RNA up-regulated in bladder carcinoma and embryo, influencing cell growth and promoting invasion. FEBS Lett. 2008; 582:1919-27. doi: 10.1016/j. febslet.2008.05.012.

10. Li F, Hu CP. Long Non-Coding RNA Urothelial Carcinoma Associated 1 (UCA1): Insight into Its Role in Human Diseases. Crit Rev Eukaryot Gene Expr. 2015; 25:191-7.

11. Cheng N, Cai W, Ren S, Li X, Wang Q, Pan H, Zhao M, Li J, Zhang Y, Zhao C, Chen X, Fei K, Zhou C, et al. Long noncoding RNA UCA1 induces non-T790M acquired resistance to EGFR-TKIs by activating the AKT/mTOR pathway in EGFR-mutant non-small cell lung cancer. Oncotarget. 2015; 6:23582-93. doi: 10.18632/oncotarget.4361.

12. Na XY, Liu ZY, Ren PP, Yu R, Shang XS. Long non-coding RNA UCA1 contributes to the progression of prostate cancer and regulates proliferation through KLF4-KRT6/13 signaling pathway. Int J Clin Exp Med. 2015; 8:12609-16.

13. Wang F, Ying HQ, He BS, Pan YQ, Deng QW, Sun HL, Chen J, Liu X, Wang SK. Upregulated IncRNA-UCA1 contributes to progression of hepatocellular carcinoma through inhibition of miR-216b and activation of FGFR1/ ERK signaling pathway. Oncotarget. 2015; 6:7899-917. doi: 10.18632/oncotarget.3219.

14. Hu JJ, Song W, Zhang SD, Shen XH, Qiu XM, Wu HZ, Gong PH, Lu S, Zhao ZJ, He ML, Fan H. HBx-upregulated lncRNA UCA1 promotes cell growth and tumorigenesis by recruiting EZH2 and repressing p27Kip1/CDK2 signaling. Sci Rep. 2016; 6:23521. doi: 10.1038/srep23521.

15. Wang XS, Zhang Z, Wang HC, Cai JL, Xu QW, Li MQ, Chen YC, Qian XP, Lu TJ, Yu LZ, Zhang Y, Xin DQ, $\mathrm{Na} Y Q$, et al. Rapid identification of UCA1 as a very sensitive and specific unique marker for human bladder carcinoma. Clin Cancer Res. 2006; 12:4851-8. doi: 10.1158/1078-0432.CCR-06-0134.

16. Tuo YL, Li XM, Luo J. Long noncoding RNA UCA1 modulates breast cancer cell growth and apoptosis through decreasing tumor suppressive miR-143. Eur Rev Med Pharmacol Sci. 2015; 19:3403-11.

17. Ni B, Yu X, Guo X, Fan X, Yang Z, Wu P, Yuan Z, Deng Y, Wang J, Chen D, Wang L. Increased urothelial cancer associated 1 is associated with tumor proliferation and metastasis and predicts poor prognosis in colorectal cancer. Int J Oncol. 2015; 47:1329-38. doi: 10.3892/ijo.2015.3109.

18. Han Y, Yang YN, Yuan HH, Zhang TT, Sui H, Wei XL, Liu L, Huang P, Zhang WJ, Bai YX. UCA1, a long non-coding RNA up-regulated in colorectal cancer influences cell proliferation, apoptosis and cell cycle distribution. Pathology. 2014; 46:396-401. doi: 10.1097/ PAT.0000000000000125.

19. Bian Z, Jin L, Zhang J, Yin Y, Quan C, Hu Y, Feng Y, Liu H, Fei B, Mao Y, Zhou L, Qi X, Huang S, et al. LncRNAUCA1 enhances cell proliferation and 5-fluorouracil resistance in colorectal cancer by inhibiting miR-204-5p. Sci Rep. 2016; 6:23892. doi: 10.1038/srep23892.

20. Li JY, Ma X, Zhang CB. Overexpression of long noncoding RNA UCA1 predicts a poor prognosis in patients with esophageal squamous cell carcinoma. Int J Clin Exp Pathol. 2014; 7:7938-44.

21. Yang Y, Jiang Y, Wan Y, Zhang L, Qiu J, Zhou S, Cheng W. UCA1 functions as a competing endogenous RNA to suppress epithelial ovarian cancer metastasis. Tumour Biol. 2016; 37:10633-41. doi: 10.1007/s13277-016-4917-1.

22. Zheng Q, Wu F, Dai WY, Zheng DC, Zheng C, Ye H, Zhou B, Chen JJ, Chen P. Aberrant expression of UCA1 in gastric cancer and its clinical significance. Clin Transl Oncol. 2015; 17:640-6. doi: 10.1007/s12094-015-1290-2.

23. Gao J, Cao R, Mu H. Long non-coding RNA UCA1 may be a novel diagnostic and predictive biomarker in plasma for early gastric cancer. Int J Clin Exp Pathol. 2015; 8:12936-42.

24. Shang C, Guo Y, Zhang J, Huang B. Silence of long noncoding RNA UCA1 inhibits malignant proliferation and chemotherapy resistance to adriamycin in gastric cancer. Cancer Chemother Pharmacol. 2016; 77:1061-7. doi: 10.1007/s00280-016-3029-3.

25. Tian Y, Zhang X, Hao Y, Fang Z, He Y. Potential roles of abnormally expressed long noncoding RNA UCA1 and Malat-1 in metastasis of melanoma. Melanoma Res. 2014; 24:335-41. doi: 10.1097/CMR.0000000000000080.

26. Nie W, Ge HJ, Yang XQ, Sun X, Huang H, Tao X, Chen WS, Li B. LncRNA-UCA1 exerts oncogenic functions in nonsmall cell lung cancer by targeting miR-193a-3p. Cancer Lett. 2016; 371:99-106. doi: 10.1016/j.canlet.2015.11.024.

27. Fang Z, Wu L, Wang L, Yang Y, Meng Y, Yang H. Increased expression of the long non-coding RNA UCA1 in tongue squamous cell carcinomas: a possible correlation with cancer metastasis. Oral Surg Oral Med Oral Pathol Oral Radiol. 2014; 117:89-95. doi: 10.1016/j.oooo.2013.09.007.

28. Torre LA, Bray F, Siegel RL, Ferlay J, Lortet-Tieulent J, Jemal A. Global cancer statistics, 2012. CA Cancer J Clin. 2015; 65:87-108. doi: 10.3322/caac.21262.

29. Xue M, Li X, Wu W, Zhang S, Wu S, Li Z, Chen W. Upregulation of long non-coding RNA urothelial carcinoma associated 1 by CCAAT/enhancer binding protein alpha contributes to bladder cancer cell growth and reduced 
apoptosis. Oncol Rep. 2014; 31:1993-2000. doi: 10.3892/ or.2014.3092.

30. Hiemer SE, Szymaniak AD, Varelas X. The transcriptional regulators TAZ and YAP direct transforming growth factor beta-induced tumorigenic phenotypes in breast cancer cells. J Biol Chem. 2014; 289:13461-74. doi: 10.1074/jbc. M113.529115.

31. Xue M, Pang H, Li X, Li H, Pan J, Chen W. Long noncoding RNA urothelial cancer-associated 1 promotes bladder cancer cell migration and invasion by way of the hsa-miR-145-ZEB1/2-FSCN1 pathway. Cancer Sci. 2016; 107:18-27. doi: 10.1111/cas.12844.

32. Li Z, Li X, Wu S, Xue M, Chen W. Long non-coding RNA UCA1 promotes glycolysis by upregulating hexokinase 2 through the mTOR-STAT3/microRNA143 pathway. Cancer Sci. 2014; 105:951-5. doi: 10.1111/cas.12461.

33. Fan Y, Shen B, Tan M, Mu X, Qin Y, Zhang F, Liu Y. Long non-coding RNA UCA1 increases chemoresistance of bladder cancer cells by regulating Wnt signaling. FEBS J. 2014; 281:1750-8. doi: 10.1111/febs.12737.

34. Wang T, Yuan J, Feng N, Li Y, Lin Z, Jiang Z, Gui Y. HsamiR-1 downregulates long non-coding RNA urothelial cancer associated 1 in bladder cancer. Tumour Biol. 2014; 35:10075-84. doi: 10.1007/s13277-014-2321-2.

35. Kamel MM, Matboli M, Sallam M, Montasser IF, Saad AS, El-Tawdi AH. Investigation of long noncoding RNAs expression profile as potential serum biomarkers in patients with hepatocellular carcinoma. Transl Res. 2016; 168:134-45.

36. Chou KC. Impacts of bioinformatics to medicinal chemistry. Med Chem. 2015; 11:218-34.

37. Liu Y, Zeng X, He Z, Zou Q. Inferring microRNA-disease associations by random walk on a heterogeneous network with multiple data sources. IEEE/ACM Trans Comput Biol Bioinform. 2016. doi: 10.1109/tcbb.2016.2550432.

38. Wang C, Wei L, Guo M, Zou Q. Computational approaches in detecting non- coding RNA. Curr Genomics. 2013; 14:371-7. doi: 10.2174/13892029113149990005.

39. Altman DG, McShane LM, Sauerbrei W, Taube SE. Reporting Recommendations for Tumor Marker Prognostic Studies (REMARK): explanation and elaboration. PLoS Med. 2012; 9:e1001216. doi: 10.1371/journal. pmed.1001216.

40. Tierney JF, Stewart LA, Ghersi D, Burdett S, Sydes MR. Practical methods for incorporating summary time-toevent data into meta-analysis. Trials. 2007; 8:16. doi: 10.1186/1745-6215-8-16.
41. Parmar MK, Torri V, Stewart L. Extracting summary statistics to perform meta-analyses of the published literature for survival endpoints. Stat Med. 1998; 17:2815-34.

42. DerSimonian R, Laird N. Meta-analysis in clinical trials. Control Clin Trials. 1986; 7:177-88.

43. Tao K, Yang J, Hu Y, Sun Y, Tan Z, Duan J, Zhang F, Yan H, Deng A. Clinical significance of urothelial carcinoma associated 1 in colon cancer. Int J Clin Exp Med. 2015; 8:21854-60.

44. Yang Z, Lu Y, Xu Q, Tang B, Park CK, Chen X. HULC and H19 Played Different Roles in Overall and DiseaseFree Survival from Hepatocellular Carcinoma after Curative Hepatectomy: A Preliminary Analysis from Gene Expression Omnibus. Dis Markers. 2015; 2015:191029. doi: 10.1155/2015/191029.

45. Zhang L, Cao X, Zhang L, Zhang X, Sheng H, Tao K. UCA1 overexpression predicts clinical outcome of patients with ovarian cancer receiving adjuvant chemotherapy. Cancer Chemother Pharmacol. 2016; 77:629-34. doi: 10.1007/s00280-016-2963-4.

46. Lu L, Shen Y, Tseng KF, Liu W, Duan H, Meng W. Silencing of UCA1, a poor prognostic factor, inhibited the migration of endometrial cancer cell. Cancer Biomark. 2016; 17:171-7. doi: 10.3233/cbm-160628.

47. Chen P, Wan D, Zheng D, Zheng Q, Wu F, Zhi Q. Long non-coding RNA UCA1 promotes the tumorigenesis in pancreatic cancer. Biomed Pharmacother. 2016; 83:1220-6. doi: 10.1016/j.biopha.2016.08.041.

48. Fu XL, Liu DJ, Yan TT, Yang JY, Yang MW, Li J, Huo YM, Liu W, Zhang JF, Hong J, Hua R, Chen HY, Sun YW. Analysis of long non-coding RNA expression profiles in pancreatic ductal adenocarcinoma. Sci Rep. 2016; 6:33535. doi: 10.1038/srep33535.

49. Liu H, Wang G, Yang L, Qu J, Yang Z, Zhou X. Knockdown of Long Non-Coding RNA UCA1 Increases the Tamoxifen Sensitivity of Breast Cancer Cells through Inhibition of Wnt/beta-Catenin Pathway. PLoS One. 2016; 11:e0168406. doi: 10.1371/journal.pone.0168406.

50. Zuo ZK, Gong Y, Chen XH, Ye F, Yin ZM, Gong QN, Huang JS. TGFbeta1-Induced LncRNA UCA1 Upregulation Promotes Gastric Cancer Invasion and Migration. DNA Cell Biol. 2017. doi: 10.1089/dna.2016.3553. 